\title{
Caracterización de biomasa residual de la región Arequipa para la producción de biocombustibles
}

\section{(Characterization of residual biomass from the Arequipa region for the production of biofuels)}

\author{
María Laura Stronguiló Leturia ${ }^{1}$, Lynet Milagros Chacón Febres ${ }^{1}$
}

\begin{abstract}
Resumen:
El objetivo del presente trabajo es seleccionar biomasa residual generada en la Región Arequipa que pueda utilizarse en la producción de biocombustibles (biodiesel, bioetanol y biogás). En cada caso, se parte de una matriz basada en productos con biomasa residual disponible en la región, provenientes del sector agrícola y pecuario, información que fue obtenida de la página web de la Gerencia Regional de Agricultura. En el proceso de selección se considerarán factores específicos de los residuos que se usarán como materias primas en la obtención de cada biocombustible. Para la producción de biodiesel es necesario partir del aceite extraído de semillas oleaginosas. En cuanto a la obtención de bioetanol, se requiere que la biomasa cuente con alto porcentaje de celulosa y con respecto a la generación de biogás se utilizarán excrementos de animales. Finalmente, mediante la caracterización de la biomasa residual, se han seleccionado las materias primas: zapallo de Camaná y palta de Arequipa para el biodiesel; cascarilla de arroz de Castilla y marlo de maíz de Arequipa para el bioetanol y estiércol de vaca y oveja de Caylloma para el biogás.
\end{abstract}

Palabras clave: biomasa residual; biocombustibles; biodiesel; bioetanol; biogás.

\begin{abstract}
:
The aim of this work is to select residual biomass from the Arequipa Region for the production of biofuels (biodiesel, bioethanol and biogas). In each case, the initial point is a matrix based on products with residual biomass available in the region, from the agricultural and livestock sectors, information that was obtained from the regional Management of Agriculture web site. Specific factors of the resudue that will be used as raw material for each biofuel production would be considered for the selection process. For the production of biodiesel it is necessary to start from the oil extracted from oilseeds. Regarding obtaining bioethanol, it requires that the residual biomass has high percent of cellulose. With regard to the generation of biogas, we will use animal droppings. Finally, the raw materials selected are: squash and avocado seeds for biodiesel, rice chaff and deseeded corncob for bioethanol and cow and sheep droppings for biogas.
\end{abstract}

Keywords: residual biomass; biofuels; biodiesel; bioethanol; biogas.

\footnotetext{
${ }^{1}$ Universidad Católica San Pablo, Arequipa - Perú ( \{mstronguilo, milagros.chacon\} @ucsp.edu.pe )
} 


\section{Introducción}

Se estima que la demanda de energía crecerá a razón de $1.8 \%$ anual, hasta el año 2030, lo que supone un incremento del 55\% sobre las necesidades energéticas globales que se tienen. Este creciente requerimiento de energía aumenta el interés por la búsqueda de energías que no contaminen el medio ambiente como lo hacen las no renovables. Por lo tanto, se trata de encontrar otras fuentes de energía disponibles en la naturaleza que ayuden a reducir la dependencia de las de origen fósil como carbón, petróleo, gas, etc. Estas energías son las renovables que, aunque se consuman, se vuelven a reponer, tales como biomasa, luz solar, viento, etc. (Roldán, 2013)

Una de las energías renovables usadas alrededor del mundo es la biomasa. Los recursos biomásicos incluyen cualquier fuente de materia orgánica, como desechos agrícolas, forestales, y animales así como basura urbana, los que se someten a modernos procesos de conversión, enfocados hacia la sustitución de combustibles fósiles. La biomasa tiene características específicas que determinan su uso: su composición física y química determina el tipo de combustible que se puede generar, el contenido de humedad posibilita la conversión energética, el porcentaje de cenizas indica la cantidad de materia sólida no combustible por kilogramo de material, el poder calorífico determina la energía disponible en la biomasa y la densidad aparente define el peso por unidad de volumen del material en el estado físico que presenta. (FOCER, 2002)

Se entiende como biomasa la materia orgánica originada en un proceso biológico, espontáneo o provocado, utilizable como fuente de energía. (RAE, 2001). El uso de biomasa como energía alternativa presenta las siguientes ventajas: no contamina el medio ambiente, favorece el desarrollo rural ya que este proceso puede ser utilizado en zonas aisladas para la obtención de biocombustibles y disminuye la dependencia energética exterior.

La biomasa a ser seleccionada depende de características específicas que son necesarias para la producción de cada biocombustible. El biodiesel es un éster que puede producirse a partir de diferentes tipos de aceites vegetales, para la obtención de los cuales es necesario el procesamiento de semillas oleaginosas, de las que se puede extraer aceite. Posteriormente, los glicéridos, mediante una reacción de transesterificación y utilizando catalizadores, se convierten en biodiesel. La soja representa el $79 \%$ del potencial de producción total de biodiesel, seguida por el girasol (17\%) y la palma aceitera (4\%). (Razo, Ludeña, \& Saucedo, 2007)

Para la producción de bioetanol la biomasa debe contener en su composición azúcar, almidón o lignocelulosa. A partir del almidón o lignocelulosa se debe aplicar un pretratamiento químico para la obtención de azúcares fermentables. Posteriormente se realizan la fermentación y destilación para luego obtener bioetanol. La principal fuente potencial para la producción de bioetanol es la caña de azúcar en casi todos los países de América Latina, ya que la disponibilidad de 
excedentes es generalizada. Por otro lado, el potencial de producción a partir de maíz, trigo y sorgo está concentrado en Argentina. (Razo, Ludeña, \& Saucedo, 2007)

Finalmente, el biogás es un combustible que se genera por las reacciones de biodegradación de la materia orgánica mediante la acción de microorganismos y otros factores en ausencia de oxígeno. La producción de biogás por descomposición anaeróbica es un modo considerado útil para tratar residuos biodegradables, ya que produce un combustible de valor, además de generar un efluente que puede aplicarse como abono energético. Todos los residuos orgánicos, por ejemplo: basura de cocina, restos vegetales y animales, aguas servidas, aserrines, virutas, excrementos, son adecuados para ser fermentados anaeróbicamente (en ausencia de oxígeno). Las bacterias consumen el carbono y el nitrógeno y como resultado se produce el biogás. (Castillo \& Tito, 2011). Mediante este trabajo se busca caracterizar la biomasa residual originaria de la región Arequipa y estudiar su potencialidad para la producción de biocombustibles. Se evaluarán sus características físicas y químicas pues determinan el tipo de combustible o subproducto energético que se puede generar.

\section{Metodología}

El primer paso para desarrollar este estudio fue la elaboración de una matriz basada en una serie de productos (que posean biomasa residual) disponibles en la región, provenientes del sector agrícola y ganadero, información que fue obtenida de la página web de la Gerencia Regional de Agricultura de Arequipa. Con el fin de seleccionar la biomasa que se usará en la obtención de cada biocombustible, se tomarán en cuenta diversos parámetros, algunos de los cuales resultarán determinantes para la elección.

Para la matriz correspondiente al biodiesel se tomaron en cuenta parámetros como el porcentaje de ácidos grasos insaturados ( $>50 \%$ ), tamaño de semilla (grande o mediana) y hectáreas cosechadas. Se fijaron estos criterios ya que de una semilla de mayor tamaño se puede extraer más cantidad de aceite, lo cual significaría una mayor producción de biodiesel con menor número de semillas. En cuanto a los ácidos grasos insaturados, se tomó como referencia una investigación que indica que para lograr una mayor producción de biodiesel el porcentaje debe ser mayor a 50\%. (González, 2012)

Como se observa en la Tabla 1, se seleccionaron las semillas de aceituna, palta y zapallo. Se preparó la Tabla 2 con los productos elegidos incluyendo las provincias de la región Arequipa en las que se cultivan. Posteriormente, se recolectarán las materias primas de las provincias seleccionadas y se realizará la extracción del aceite de las semillas para determinar el perfil de sus ácidos grasos, mediante análisis cromatográfico. 
Tabla 1. Materia prima seleccionada para la producción de biodiesel

\begin{tabular}{|c|c|c|c|c|c|}
\hline Producto & ha & $\begin{array}{l}\text { Ácidos grasos } \\
\text { insaturados, \% }\end{array}$ & $\begin{array}{c}\text { Tamaño de la } \\
\text { semilla }\end{array}$ & $\begin{array}{c}\text { Ácidos grasos } \\
\text { insaturados, }>\mathbf{5 0 \%}\end{array}$ & $\begin{array}{c}\text { Tamaño de semilla, } \\
\text { grande o mediana }\end{array}$ \\
\hline Palta & 196 & 74,80 & Grande & $x$ & $x$ \\
\hline Aceituna & 11 & 81,80 & Mediana & $x$ & $x$ \\
\hline Zapallo & 1209 & 55,11 & Mediana & $x$ & $x$ \\
\hline Avena & 448 & 84,53 & Pequeña & $x$ & \\
\hline Tuna & 5 & 84,00 & Pequeña & $x$ & \\
\hline Quinua & 7056 & 83,47 & Pequeña & $x$ & \\
\hline Tomate & 701 & 81,00 & Pequeña & $x$ & \\
\hline Uva & 97 & 79,16 & Pequeña & $x$ & \\
\hline Trigo & 1761 & 61,70 & Pequeña & $x$ & \\
\hline Haba & 1017 & 13,00 & Pequeña & $x$ & \\
\hline
\end{tabular}

Para el sector agrícola, las provincias se seleccionaron teniendo en cuenta el área cultivada y la ubicación geográfica del producto. (Escalante, Orduz, \& Zapata, 2011). En la Tabla 2 aparece la producción (en toneladas) de aceituna, zapallo y palta en todas las provincias de Arequipa. Se seleccionarán las muestras que tienen una mayor producción para posteriormente analizarlas y conocer su perfil de ácidos grasos.

Tabla 2. Producción (t) de las materias primas seleccionadas para la obtención de biodiesel

\begin{tabular}{|c|c|c|c|}
\hline Provincia & Aceituna & Zapallo & Palta \\
\hline Arequipa & 0,00 & 1219,17 & 8188,20 \\
\hline Camaná & 287,35 & 11054,00 & 0,00 \\
\hline Caravelí & 28205,07 & 0,00 & 2480,92 \\
\hline Castilla & 0,00 & 91,32 & 278,16 \\
\hline Caylloma & 0,00 & 48210,70 & 2584,24 \\
\hline Islay & 1246,65 & 2444,56 & 0,00 \\
\hline La Unión & 0,00 & 12,80 & 9,40 \\
\hline Condesuyos & 0,00 & 410,97 & 44,51 \\
\hline
\end{tabular}

Como se puede observar en la Tabla 2, la aceituna seleccionada es la proveniente de Caravelí, el zapallo procedente de Camaná y Caylloma y la palta de Arequipa, Caravelí y Caylloma.

Para elegir la biomasa residual para desarrollar las pruebas para la obtención del bioetanol se realizo una matriz que incluyo los productos que tienen residuos cultivados en la región Arequipa, obtenidos durante la campaña agrícola 2013 - 2014, publicados en la página web de la Gerencia Regional de Agricultura. Puesto que se decidió trabajar con materiales lignocelulósicos, se buscó su contenido teórico de celulosa. Los valores se registraron en la Tabla 3, que aparece a continuación: 
Tabla 3. Materia prima seleccionada para la producción de bioetanol

\begin{tabular}{|c|c|c|c|c|c|}
\hline Producto & $\begin{array}{c}\text { Tipo de } \\
\text { residuo }\end{array}$ & $\begin{array}{c}\text { Usos en } \\
\text { alimentación }\end{array}$ & Celulosa, \% & Producción, $\mathbf{t}$ & $\begin{array}{c}\text { Rendimiento de } \\
\text { etanol (I/t de } \\
\text { materia seca) }\end{array}$ \\
\hline Caña de azúcar & Bagazo & Ninguno & 50 & 96992,87 & 280 \\
\hline Arroz & Cascarilla & Ninguno & 39,05 & 266039 & 280 \\
\hline Maíz & Marlo & Ninguno & 36,4 & 562428,3 & 290 \\
\hline Cebada & Paja & Té o infusión & 44 & 4420,55 & 310 \\
\hline Avena & Paja & Té o infusión & 41,2 & 7882,12 & 260 \\
\hline Arroz & Paja & Para rumiantes & 34 & 266039,03 & 280 \\
\hline Trigo & Pajilla & Ninguno & 33 & 15168,41 & 290 \\
\hline
\end{tabular}

Como se observa en la Tabla 3, las materias primas seleccionadas para la producción de bioetanol son el bagazo de caña azúcar, la cascarilla de arroz y el marlo de maíz, teniendo en cuenta su porcentaje de celulosa y el hecho que sus residuos no se utilizan como alimento.

La Tabla 4 muestra las provincias de la región Arequipa donde se cosechan dichos productos y la cantidad producida en toneladas. Se seleccionarán aquellas de mayor producción para analizar el porcentaje de celulosa presente.

Tabla 4. Producción (t) de las materias primas seleccionadas para la obtención de bioetanol

\begin{tabular}{|c|c|c|c|}
\hline Provincia & Caña de azúcar & Arroz & Maíz \\
\hline Arequipa & 0,00 & 0,00 & 191695,08 \\
\hline Camaná & 0,00 & 109718,34 & 2773,54 \\
\hline Caravelí & 0,00 & 0,00 & 0,00 \\
\hline Castilla & 0,00 & 90007,89 & 19209,70 \\
\hline Caylloma & 0,00 & 0,00 & 281191,00 \\
\hline Islay & 10400,91 & 65943,20 & 67558,95 \\
\hline La Unión & 0,00 & 0,00 & 0,00 \\
\hline Condesuyos & 0,00 & 369,60 & 0,00 \\
\hline
\end{tabular}

Como se ha señalado en la Tabla 4, se analizará el bagazo de caña de azúcar proveniente de Islay, la cascarilla de arroz procedente de Camaná y Castilla y el marlo de maíz de Arequipa y Caylloma. Estas muestras se enviarán a un laboratorio externo para determinar el contenido de celulosa. Se seleccionarán las que resulten con un porcentaje mayor de celulosa para emplearlas en la producción de bioetanol.

Para la producción del biogás se utilizarán desechos animales, que son principalmente generados del sector pecuario (estiércol). Las muestras se someterán al proceso de biodigestión anaerobia, 
del cual se obtienen compuestos altamente energéticos que pueden usarse para producir energía eléctrica y calorífica.

En la tabla 5 aparecen las especies de las que se puede obtener estiércol. La información procede de la página web de la Gerencia Regional de Agricultura de Arequipa. Se ha añadido el estiércol de cuy teniendo en cuenta que en la región existe la Asociación de Criadores de Cuyes. Los datos se complementan con los valores teóricos del contenido de carbono y nitrógeno y la relación $\mathrm{C} / \mathrm{N}$. El carbono es una fuente de energía para los microorganismos y el nitrógeno es un elemento necesario para la síntesis proteica. La relación $\mathrm{C} / \mathrm{N}$ es un índice significativo de la digestibilidad de un determinado material orgánico. (Escalante, Orduz, \& Zapata, 2011)

Tabla 5. Materia prima seleccionada para la producción de biogás

\begin{tabular}{|c|c|c|c|c|c|}
\hline Estiércol de & $\begin{array}{c}\text { Carbono, \% } \\
\text { peso }\end{array}$ & $\begin{array}{c}\text { Nitrógeno, \% } \\
\text { peso }\end{array}$ & Relación C/N & $\begin{array}{c}\text { Contenido } \\
\text { seco, \% }\end{array}$ & $\begin{array}{c}\text { Contenido } \\
\text { hídrico, \% }\end{array}$ \\
\hline Oveja & 16 & 0,55 & 29 & 24 & 76 \\
\hline Vaca & 7,3 & 0,29 & 25 & 17 & 83 \\
\hline Cuy & 39 & 1,9 & 20 & 70 & 30 \\
\hline Cerdo & 17,4 & 1,1 & 16 & 18 & 82 \\
\hline Ave & 41 & 1,3 & 32 & 16 & 84 \\
\hline Cabra & 39,8 & 2,2 & 18 & 32 & 68 \\
\hline Alpaca & 48 & 3,6 & 13 & 37 & 63 \\
\hline Llama & 55,4 & 3,93 & 14 & 38 & 62 \\
\hline Vicuña & 50,3 & 3,62 & 14 & 35 & 65 \\
\hline
\end{tabular}

La formación de metano y ácido puede darse con valores mínimos de C/N de 16. Sin embargo, las bacterias responsables del proceso pueden tener un excelente desempeño cuando el residuo presenta una relación C/N entre 25 y 30. (Escalante, Orduz, \& Zapata, 2011). Según la Tabla 5, se ha seleccionado el estiércol de oveja, vaca y cuy.

Con estas materias primas se podrá evaluar diferentes relaciones de $\mathrm{C} / \mathrm{N}$ y su posterior influencia en el biogás. En el sector pecuario, los criterios empleados para definir las unidades de muestreo (provincias) fueron: la cantidad de población animal y la ubicación geográfica y climatológica. (Escalante, Orduz, \& Zapata, 2011)

En la Tabla 6 se ha registrado la cantidad de cabezas de vacas, ovejas y cuyes en las diferentes provincias de la región Arequipa. Se seleccionaron las provincias donde se cría mayor cantidad de cabezas de cada especie.

Como se puede observar en los datos de la tabla 6, la mayor cantidad de ganado seleccionado se encuentra en Caylloma y Arequipa. Se recolectarán muestras de estiércol de cuy, vaca y oveja de estas provincias y se enviarán a un laboratorio externo para determinar la relación C/N. 
Tabla 6. Cantidad de ganado (cabezas) de las especies seleccionadas para la producción de biogás

\begin{tabular}{|c|c|c|c|}
\hline Provincia & Vaca & Oveja & Cuy \\
\hline Arequipa & 55673 & 26971 & 116803 \\
\hline Camaná & 4757 & 3588 & 12611 \\
\hline Caravelí & 8456 & 8119 & 21833 \\
\hline Castilla & 32731 & 17616 & 35613 \\
\hline Caylloma & 80686 & 56885 & 159903 \\
\hline Islay & 12170 & 3961 & 24574 \\
\hline La Unión & 16685 & 15766 & 37901 \\
\hline Condesuyos & 15475 & 18021 & 27652 \\
\hline
\end{tabular}

Como ya se tiene definida que materia prima se va a evaluar experimentalmente, se desarrollaron pruebas en laboratorio externos para su caracterización y selección final de la biomasa residual que tiene potencial de ser utilizada para la producción de biocombustibles.

\section{Resultados y Discusión}

\section{Selección de la materia prima para biodiesel}

En la parte teórica se selecciono el zapallo procedente de Camaná y Caylloma y la palta de Arequipa, Caravelí y Caylloma. Antes de realizar el análisis cromatográfico, se extrajo el aceite contenido en las semillas mediante el siguiente procedimiento:

- Secado de semillas: La materia prima se almacena en un lugar fresco, seco y ventilado para evitar la formación de hongos y mohos. Se seca al ambiente por $5-6$ días durante $6-7$ horas cada día. Seguidamente, pasa a ser triturada en un molino industrial, hasta obtener un polvo casi homogéneo. Se coloca la muestra molida en una cápsula de porcelana y se seca en una estufa durante 4 horas a una temperatura de $70^{\circ} \mathrm{C}$.

- Extracción del aceite: Para esto, se pesa 10 gramos de la muestra seca y molida directamente en un cartucho de papel filtro rápido. Se coloca $125 \mathrm{ml}$ de $\mathrm{n}$-hexano en un balón de fondo plano y se arma el equipo Soxhlet, como aparece en la Figura 1. Luego se inicia la extracción a una temperatura de $70^{\circ} \mathrm{C}$, manteniendo el goteo del solvente a una velocidad mayor a 150 gotas por minuto, durante 6 horas. Cumplido el tiempo fijado, se deja enfriar el sistema. 


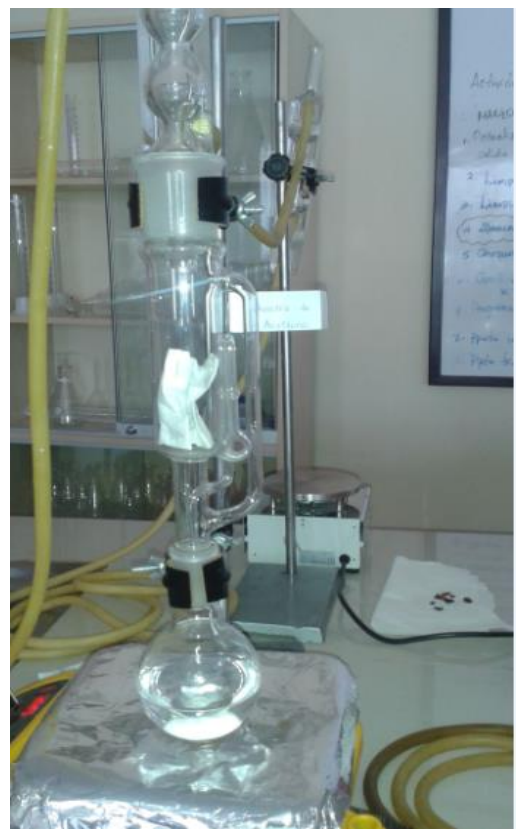

Figura 1. Extracción del aceite por el método Soxhlet

- Destilación: Posteriormente, se desarma el equipo y se procede a destilar el hexano a una temperatura de $65^{\circ} \mathrm{C}$, como se observa en la Figura 2.

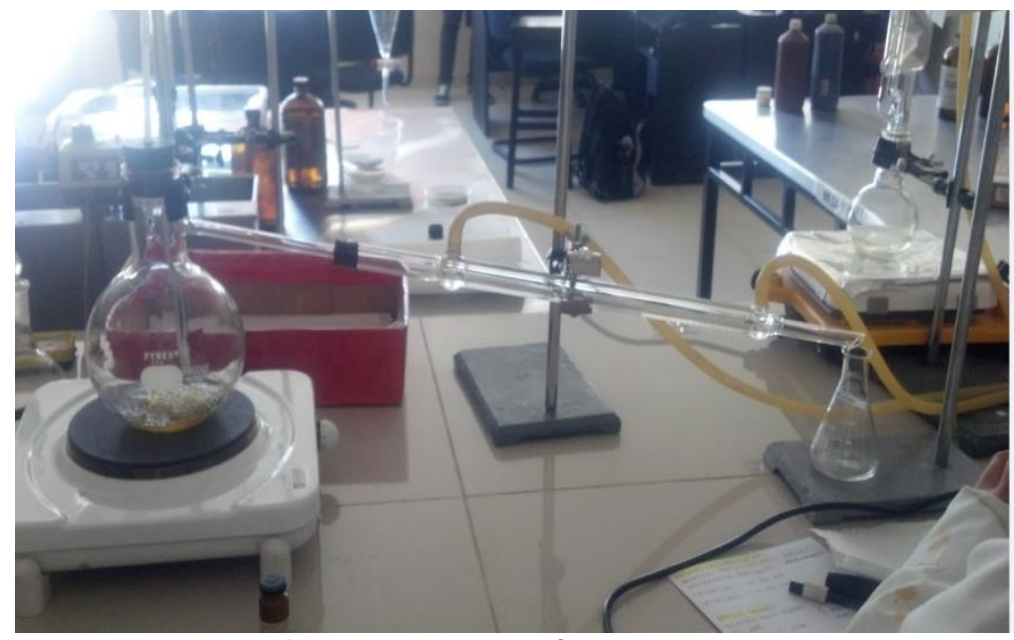

Figura 2. Destilación del hexano

Finalmente, la muestra del aceite extraído ( $2 \mathrm{ml}$ aproximadamente) se vierte en un vial y se envía a un laboratorio externo para la determinación del perfil de sus ácidos grasos. Los resultados obtenidos se resumen en la Tabla 7.

Un factor determinante para la selección de materia prima es el porcentaje de ácidos grasos saturados debido a que dificultan la reacción de transesterificación, que es importante para la obtención de biodiesel pues reduce la alta viscosidad de los triglicéridos. (Meher \& Vidya Sagar \& Naik, 2006). Según los resultados mostrados en la Tabla 7, se seleccionan la palta de la provincia de Arequipa y el zapallo de Camaná. 
Tabla 7. Porcentaje de ácidos grasos saturados en las muestras de aceite

\begin{tabular}{|c|c|c|}
\hline Muestra & Procedencia & Ácidos grasos saturados, \% \\
\hline Palta & Arequipa & 5,84 \\
\hline Palta & Caravelí & 6,90 \\
\hline Palta & Caylloma & 9,51 \\
\hline Zapallo & Camaná & 10,89 \\
\hline Zapallo & Caylloma & 13,35 \\
\hline Aceituna & Caravelí & 11,14 \\
\hline
\end{tabular}

Selección de la materia prima para bioetanol

Para la selección de la materia prima para el bioetanol se analizará el bagazo de caña de azúcar proveniente de Islay, la cascarilla de arroz procedente de Camaná y Castillla y el marlo de maíz de Arequipa y Caylloma. La cantidad de materia prima que se requiere para los ensayos es $50 \mathrm{~g}$. Luego de recolectarla se almacenó temporalmente dentro del laboratorio, se limpió para eliminar impurezas y se dejó orear para evitar la formación de hongos. Para realizar los análisis de laboratorio la materia prima debía estar seca, por lo que se dejó secar al sol durante 5 días, aproximadamente 6 - 7 horas al día. Las muestras secas se enviaron a un laboratorio de análisis para determinar el contenido de celulosa.

A continuación, la Tabla 8 detalla los resultados que reportó el laboratorio externo:

Tabla 8. Porcentaje de celulosa presente en los materiales lignocelulósicos

\begin{tabular}{|c|c|c|c|}
\hline Muestra & Residuo & Procedencia & Celulosa, \% \\
\hline Arroz & Cascarilla & Castilla & 52,80 \\
\hline Arroz & Cascarilla & Camaná & 50,69 \\
\hline Maíz & Marlo & Arequipa & 27,35 \\
\hline Caña de azúcar & Bagazo & Islay & 26,04 \\
\hline Maíz & Marlo & Caylloma & 20,16 \\
\hline
\end{tabular}

Según la Tabla 8, las materias primas seleccionadas para realizar las pruebas experimentales de obtención de bioetanol son la cascarilla de arroz de la provincia de Castilla y el marlo del maíz de Arequipa.

\section{Selección de la materia prima para biogas}

Según el levantamiento de información se recolectará muestras de estiércol de cuy, vaca y oveja de las provincias de Caylloma y Arequipa. Los resultados obtenidos se encuentran en la Tabla 9.

En base a los valores reportados para la relación $\mathrm{C} / \mathrm{N}$, se seleccionó el estiércol de vaca y oveja de la provincia de Caylloma. Con esas dos materias primas se realizarán pruebas experimentales de obtención de biogás, para ver cuál de ellas produce un biocombustible de mejor calidad. 
Como se observa en las tablas 1, 3 y 5, en este estudio se evaluaron cinco tipos de biomasa residual, de los cuales cuatro pertenecen al sector agrícola (semillas, cascarilla, bagazo y marlo) y uno al sector pecuario (estiércol). La caracterización de la biomasa residual agrícola y pecuaria contempló la determinación de algunos de los parámetros que describen la composición de la materia orgánica correspondientes al análisis físico químico.

Tabla 9. Relación $\mathrm{C} / \mathrm{N}$ de las muestras de estiércol

\begin{tabular}{|c|c|c|c|c|}
\hline Estiércol de & Procedencia & Carbono, \% & Nitrógeno, \% & Relación C/N \\
\hline Vaca & Caylloma & 31,63 & 0,94 & 33,65 \\
\hline Oveja & Caylloma & 29,52 & 1,19 & 24,81 \\
\hline Cuy & Arequipa & 56,08 & 2,60 & 21,57 \\
\hline Oveja & Arequipa & 35,62 & 2,32 & 15,35 \\
\hline Cuy & Caylloma & 35,25 & 2,62 & 13,45 \\
\hline Vaca & Arequipa & 17,98 & 1,51 & 11,91 \\
\hline
\end{tabular}

En las materias primas que se utilizarán para producir biodiesel esto implica realizar un análisis proximal que abarca determinar en las muestras el contenido de humedad, cenizas y materia volátil así como el perfil de ácidos grasos (ver tablas 10 y 11, respectivamente).

Tabla 10. Análisis proximal de las materias primas seleccionadas para la obtención de biodiesel (semillas

\begin{tabular}{|c|c|c|c|c|}
\hline Muestra & Procedencia & Humedad, \% & Cenizas, \% & Materia volátil, \% \\
\hline Zapallo & Camaná & 50,20 & 2,39 & 47,41 \\
\hline Palta & Arequipa & 56,60 & 1,16 & 42,24 \\
\hline Aceituna & Caravelí & 25,45 & 54,00 & 20,55 \\
\hline
\end{tabular}

Los resultados obtenidos muestran una humedad alta para las semillas de zapallo y palta, lo que las inclinaría a ser aprovechadas energéticamente mediante un proceso bioquímico. Además, el alto contenido de cenizas en las semillas de aceituna es perjudicial para su aprovechamiento por vías térmicas ya que reduce su poder calorífico. Por lo tanto, el perfil de los ácidos grasos (ver Tabla 11) será el factor que determine la selección ya que las propiedades combustibles del biodiesel dependen de la cantidad de cada ácido graso presente en la materia prima. (Karmakar, 2010).

En consecuencia, las materias primas seleccionadas para la producción de biodiesel son las semillas de palta de Arequipa y zapallo de Camaná, ya que son las que resultaron con un porcentaje menor de ácidos grasos saturados.

En cuanto a los materiales lignocelulósicos seleccionados para la obtención de bioetanol, se desea valorar la aplicación industrial de sus residuos como fuente energética, por lo que además del análisis proximal se realizó el estructural, que incluye la determinación del contenido de celulosa (ver tablas 12 y 6 , respectivamente). 
Tabla 11. Perfil de ácidos grasos (\%) por CG-FID de las materias primas seleccionadas para la obtención

\begin{tabular}{|c|c|c|c|c|c|c|}
\hline Procedencia & Caravelí & Arequipa & Caylloma & Camaná & Caylloma & Caravelí \\
\hline $\begin{array}{c}\text { Tipo de } \\
\text { ácido graso }\end{array}$ & Palta & Palta & Palta & Zapallo & Zapallo & Aceituna \\
\hline Esteárico & 0,36 & 1,21 & 0,75 & 2,35 & 4,65 & 1,98 \\
\hline Palmítico & 6,54 & 4,63 & 8,76 & 8,54 & 8,70 & 9,16 \\
\hline Linoleico & 22,24 & 14,00 & 3,31 & 39,32 & 39,52 & 9,50 \\
\hline Palmitoleico & 4,21 & 5,62 & 5,20 & - & - & - \\
\hline Oleico & 65,30 & 73,41 & 78,45 & 47,58 & 45,78 & 78,16 \\
\hline Otros & 1,35 & 1,13 & 3,53 & 2,21 & 1,35 & 1,20 \\
\hline
\end{tabular}

Tabla 12. Análisis proximal de las materias primas seleccionadas para la obtención de bioetanol.

\begin{tabular}{|c|c|c|c|c|c|}
\hline Producto & Residuo & Procedencia & Humedad, \% & Cenizas, \% & Materia volátil, \% \\
\hline $\begin{array}{c}\text { Caña de } \\
\text { azúcar }\end{array}$ & Bagazo & Islay & 66,10 & 0,87 & 33,03 \\
\hline Maíz & Marlo & Arequipa & 10,27 & 3,97 & 85,76 \\
\hline Arroz & Cascarilla & Castilla & 8,54 & 19,98 & 71,48 \\
\hline
\end{tabular}

La humedad del bagazo lo inclina a ser aprovechado energéticamente mediante un proceso bioquímico como la fermentación o la digestión anaerobia. Sin embargo, en la selección influyó mas el hecho que la producción de bioetanol alcanza elevados rendimientos cuando se utiliza como sustrato una biomasa que posee un alto contenido de celulosa. (Escalante, Orduz, \& Zapata, 2011). Por lo tanto, las materias primas elegidas para la producción de bioetanol son la cascarilla de arroz de Castilla y el marlo del maíz de Arequipa.

A las muestras de estiércol que se utilizarán para producir biogás se les determinó la relación $\mathrm{C} / \mathrm{N}$ (ver Tabla 9). Los resultados obtenidos determinaron que las materias primas seleccionadas para la producción de biogás sean las muestras de estiércol de vaca y oveja procedentes de Caylloma.

Ambos residuos (estiércol vacuno y ovino) pueden tener un aprovechamiento energético mediante bioprocesos, como la digestión anaerobia, ya que sus características físico químicas (ver Tabla 13: altos porcentajes de materia volátil y orgánica) los hacen idóneos para el crecimiento y desarrollo de consorcios microbianos capaces de degradar la materia orgánica y producir biogás (Escalante, Orduz, \& Zapata, 2011). 
Tabla 13. Análisis físico químico de las muestras de estiércol seleccionadas para la obtención de biogás

\begin{tabular}{|c|c|c|}
\hline Análisis & Vacuno & Ovino \\
\hline Sólidos totales, \% & 41,80 & 59,20 \\
\hline Materia volátil, \% & 59,00 & 43,15 \\
\hline Humedad, \% & 58,20 & 40,80 \\
\hline Ceniza, \% & 13,04 & 30,20 \\
\hline Materia orgánica, \% & 86,96 & 69,80 \\
\hline Nitrógeno, \% & 1,16 & 1,27 \\
\hline pH & 8,00 & 8,00 \\
\hline Relación C/N & 43,48 & 31,88 \\
\hline
\end{tabular}

\section{Conclusiones y Recomendaciones}

Tomando en consideración la influencia del perfil de ácidos grasos en las propiedades combustibles del biodiesel, se han seleccionado como materias primas para su obtención las semillas de palta de Arequipa y zapallo de Camaná.

Teniendo en cuenta su porcentaje de celulosa, las materias primas que se seleccionaron para realizar las pruebas de obtención de bioetanol son la cascarilla de arroz de la provincia de Castilla y el marlo del maíz de Arequipa.

La producción de biocombustibles de segunda generación se basa en la lignocelulosa, pues ofrece la posibilidad de usar aquellas partes de las plantas o cultivos que no tienen valor para la alimentación humana.

A partir de los resultados obtenidos en la determinación de la relación $\mathrm{C} / \mathrm{N}$, se seleccionaron las muestras de estiércol de vaca y oveja de la provincia de Caylloma para realizar las pruebas experimentales de producción de biogás y ver cuál de ellas produce un biocombustible de mejor calidad.

El contenido de sólidos volátiles orgánicos (grasas, carbohidratos y proteínas) representa el material disponible para el desarrollo y reproducción de bacterias anaeróbicas, y ha sido un factor determinante en la selección de las excretas bovinas y ovinas para la producción de biogás.

\section{Agradecimiento}

Las autoras agradecen al Programa Nacional de Innovación para la Competitividad y Productividad por el financiamiento del presente trabajo, realizado como parte del proyecto identificado con contrato no $151-$ FINCyT-IB-2013. 


\section{Bibliografía}

Castillo, D., \& Tito, C. (2011). Obtención de biogás a partir de excremento de cuy en condiciones ambientales en Tacna, Perú. Ciencia \& Desarrollo, 13-20.

Escalante, H., Orduz, J., \& Zapata, H. (2011). Atlas del Potencial Energético de la Biomasa Residual en Colombia. . En Anexo B: Muestreo y caracterización de la biomasa residual en Colombia (págs. 131-136). Colombia.

FOCER. (2002). Biomasa. Manual sobre energía renovable. Costa Rica: BUN-CA.

Karmakar, A. K. (2010). Properties of various plants and animals feedstocks for biodiesel production. Bioresource Technology, 101-7202.

Meher, L., \& Vidya Sagar \& Naik, S. (2006). Technical aspects of biodiesel production by transesterification - a review. Renewable \& Sustainable Energy Reviews, 10-251.

RAE. (2001). Diccionario de la lengua española. España.

Razo, C., Ludeña, C., \& Saucedo, A. (2007). Producción de biomasa para biocombustibles líquidos: El potencial de América Latina y el Caribe. Santiago de Chile: CEPAL.

Roldán, J. (2013). Energías Renovables. Lo que hay que saber. Madrid: Ediciones Paraninfo. 